Archives

9| 1992

Varia

\title{
Le saint illettré dans l'hagiographie islamique
}

\section{Michel Chodkiewicz}

\section{(apenEdition}

\section{Journals}

Édition électronique

URL : http://journals.openedition.org/ccrh/2799

DOI : $10.4000 /$ ccrh. 2799

ISSN : $1760-7906$

Éditeur

Centre de recherches historiques - EHESS

Édition imprimée

Date de publication : 15 avril 1992

ISSN : 0990-9141

Référence électronique

Michel Chodkiewicz, "Le saint illettré dans I'hagiographie islamique », Les Cahiers du Centre de Recherches Historiques [En ligne], 9| 1992, mis en ligne le 18 mars 2009, consulté le 19 avril 2019. URL : http://journals.openedition.org/ccrh/2799 ; DOI : 10.4000/ccrh.2799

Ce document a été généré automatiquement le 19 avril 2019

Article L.111-1 du Code de la propriété intellectuelle. 


\title{
Le saint illettré dans l'hagiographie islamique
}

\author{
Michel Chodkiewicz
}

Laqad kâna lakum fî rasûli Llâhi uswatun hasana, «Certes, il y a pour vous dans l'Envoyé de Dieu un modèle excellent » (Cor. 33 : 21) : ce verset coranique institue sans ambiguïté le paradigme auquel devra faire référence en islam toute forme concevable de perfection. Cette référence - si abondamment invoquée dans les écrits et les propos - peut être l'expression d'une foi sincère. Elle peut aussi, bien sûr, n'être qu'un simple gage de conformité voilant des intérêts, des calculs ou des peurs. Mais il reste qu'on ne peut comprendre l'histoire des sociétés islamiques sans prendre en considération le rôle central qui est le sien dans la constitution des normes individuelles et communautaires et dans la définition de l'idéal auquel ces normes sont ordonnées: car cette imitatio Prophetae, sous quelque aspect qu'elle se présente, est toujours une asymptote. Elle ne peut que tendre, sans jamais l'atteindre, à la plénitude insurpassable du «modèle excellent ».

2 Pour la âmma-pour le commun des croyants-cette imitation gardera souvent un caractère relativement extérieur : au-delà du respect des formes légales qui découlent de la pratique ou des propos du Prophète et s'imposent à tous, le pieux musulman s'efforcera, entre plusieurs comportements également licites, de choisir celui pour lequel le Prophète a marqué une préférence et donc de privilégier par exemple certains gestes, certains vêtements, certaines nourritures. Mais Muhammad a été envoyé par Dieu pour " parfaire » les makârim al-akhlâq, les «nobles caractères » ou les «nobles vertus " ${ }^{1}$ et $l^{\prime}$ imitatio Prophetae ne saurait se limiter à une scrupuleuse observance des conduites apparentes dont la tradition livre l'exemple. Elle doit viser aussi, dans la mesure du possible, à conformer l'être intérieur du croyant au modèle prophétique. De ce principe découle, non pas à proprement parler un système de valeurs éthiques - ces dernières se trouvent déjà énoncées dans le Coran - mais un mode de représentation de ces valeurs, fondé sur l'existence historique d'un homme qui les incarne.

3 Cette forme d'adhésion à la uswa hasana, cependant, n'épuise pas les virtualités du modèle muhammadien. De manière plus ou moins explicite selon les auteurs, les maîtres 
spirituels - et pas seulement ceux qui appartiennent à des époques tardives - vont élaborer une doctrine qui identifie le Prophète à l'Insân kâmil, à l'Homme Parfait, nuskhat al-haqq, « image de Dieu ", cause finale de la création toute entière. Je ne m'étendrai pas sur cette notion et sur toutes celles qui lui sont connexes - celles entre autres de nûr muhammadî ("Lumière muhammadienne») ou de haqîqa muhammadîyya ("Réalité muhammadienne »). L'exploration en a été entreprise depuis longtemps et bien qu'elle soit, à mon avis, loin d'être terminée - nombre de textes fondamentaux n'ont pas été analysés de façon suffisamment pénétrante - des travaux comme ceux de Nicholson, de Toshihiko Izutsu ou, plus récemment, d'un jeune compatriote de ce dernier, Masataka Takeshita, dessinent assez clairement les grandes lignes de cette anthropologie sacrée ${ }^{2}$. L' insân kâmil est, selon la formule d'Ibn Arabî, l'« isthme » (barzakh) entre Dieu et l'univers', il «conjoint les réalités divines, c'est à dire les Noms (de Dieu), et les réalités créaturelles $»^{4}$; il est la Parole totalisatrice (al-kalima aljâmi'a) ${ }^{5}$ qui contient toutes les Paroles de Dieu, c'est à dire tous les êtres ${ }^{6}$. La " perfection» dont il s'agit ici ne doit pas être comprise en un sens simplement moral, bien qu'elle implique nécessairement mais à titre subordonné l'excellence des vertus: elle est de nature métaphysique et, même si tout membre du genre humain a en principe vocation à ce statut éminent, elle ne peut être véritablement assignée qu'à celui en qui s'accomplit intégralement le théomorphisme originel de l'homme que Dieu a créé "selon Sa Forme" ainsi que l'énonce un hadîth souvent cité 7 . Ce miroir sans défaut en lequel Dieu Se contemple ${ }^{8}$ 'est autre, stricto sensu, que le Prophète lui-même ${ }^{9}$. Mais, en ce « dernier tiers de la nuit » où l'univers est entré, à la mort de Muhammad ${ }^{10}$, l'insân kâmil reste cependant présent jusqu'à la consommation des siècles en la personne des awliyâ, des saints (singulier walî), qui sont les héritiers (wurathâ) du Prophète ${ }^{11}$.

Deux questions préliminaires exigent une réponse, qui sera forcément succinte. La première concerne l'emploi du mot «saint» pour traduire walî. Un de mes collègues britanniques a critiqué très vivement cet usage dans un livre récent ${ }^{12}$. Cette traduction est assurément discutable : étymologiquement, d'abord, parce que ces deux mots renvoient à des valeurs sémantiques diamétralement opposées: transcendance, inaccessibilité pour sanctus, proximité pour walî. Doctrinalement ensuite: la conception islamique de la nature de la walâya n'est pas identique en tous points, il s'en faut, à la conception chrétienne de la nature de la sainteté. Certains saints chrétiens ne seraient pas en islam considérés comme des awliyâ et la proposition inverse est sans doute également valable. Il n'en demeure pas moins que les représentations du saint et du walî et la manière dont leurs fonctions dans l'économie du sacré sont comprises présentent assez de similitudes pour légitimer un usage ancien et commode.

D'autre part, lorsqu'on parle de saints en islam, de qui parle-t-on? L'historien de la chrétienté peut définir approximativement le groupe d'individus auquel il s'intéresse en faisant l'addition des saints canonisés et de ceux qui, sans l'être, ont été l'objet d'une vénération attestée. Pour l'islam, nous n'avons pas cette ressource: pas de sanctoral officiel-et, quant au recensement des "cultes " populaires, il est très largement lacunaire. Je retiens donc provisoirement un critère intellectuellement peu rigoureux mais pratique : la « canonisation » par la littérature. Sont saints les personnages identifiés comme tels par la tradition hagiographique - et plus particulièrement, ceux dont les noms reviennent toujours dans les grandes compilations. Beaucoup d'hagiographes ayant été eux-mêmes canonisés par les hagiographes postérieurs, il n'est pas interdit de voir là, jusqu'à un certain point, un système de cooptation : quoiqu'il en soit, n'oublions pas que 
même la liste la plus extensive à ma connaissance - celle de Nabhânî, mort en 1931, qui dans son Jâmi karamât al-awliyâ ${ }^{13}$ a recensé quelque mille quatre cents awliyâa - ne représente en fait qu'un échantillon limité de l'immense tribu des saints.

Bien que l'hagiographie nous donne souvent l'impression contraire - on a parfois le sentiment que, sous des noms différents, c'est le même walî qui revient, dans un décor à peine modifié, avec des karamât qui semblent empruntées à un magasin d'accessoires assez peu fourni - il n'y a rien de plus singulier que le saint. J'ai parlé de tribu, mais c'est une tribu dont chaque membre puise dans un pool génétique immense et divers - la surabondante réalité muhammadienne. Reste qu'on peut tout de même procéder à des regroupements, observer la récurrence de certains types dont chaque saint représente une variante ${ }^{14}$. Je ne tenterai pas ici de les identifier. Mais il est, parmi eux, un cas sur lequel je crois devoir insister, non seulement en raison de sa fréquence mais parce qu'il met en évidence l'aspect spécifique de la perfection muhammadienne » qui est commun à tous les modèles de sainteté, si divers soient-ils.

7 L'illustre théologien Fakhr al-dîn Râzî vint un jour trouver un saint non moins illustre - il s'agissait de Najm al-dîn Kubrâ - et demanda à entrer dans la Voie sous sa direction. Kubrâ chargea son disciple d'installer Râzî dans une cellule et prescrivit au théologien de s'adonner dans cette khalwa à l'invocation. Il ne s'en tint cependant pas là : projetant sur Râzî son énergie spirituelle, son tawajjuh, il le dépouilla, nous dit-on, de toutes les sciences livresques qu'il avait acquises. Or, quand Râzî prit conscience que s'effaçait soudainement de sa mémoire les connaissances dont il était si fier, il se mit à crier de toutes ses forces : « Je ne peux pas, je ne peux pas ». L'expérience s'arrêta là. Râzî sortit de sa khalwa et prit congé de Najm al-dîn Kubrâ ${ }^{15}$.

8 Ce détour s'imposait pour éclairer, a contrario, le cas du saint ummî. Ce dernier mot, habituellement traduit par « illettré », apparait plusieurs fois dans le Coran, au singulier, pour qualifier le Prophète lui-même et, au pluriel, pour désigner les membres de la communauté vers laquelle il est envoyé. Je ne tenterai pas ici l'exégèse de ces versets, qui nous entraînerait trop loin. Je n'insisterai pas non plus sur l'élaboration doctrinale de la notion de ummiyya, d'«illettrisme ", chez les maîtres du tasawwuf: Ibn Arabî, entre autres, explique clairement, dans le chapitre deux-cent quatre vingt-neuf des Futûhât, que l'on peut être ummî sans être analphabète dès lors que l'intellect est capable de suspendre ses opérations et qu'à l'exemple du Prophète, récepteur virginal de la Révélation, l'être s'ouvre tout entier aux lumières de la grâce : en ce sens, d'ailleurs, on peut dire que tout walî est ummî. Il est à noter, au surplus, pour démentir l'idée selon laquelle une activité « intellectuelle » serait contradictoire avec cette disposition à accueillir une illumination surnaturelle, qu'un autre grand maître de la lignée du Shaykh al-Akbar, lui-même un saint, Abd al-karîm al-jîlî, insiste sur l'importance des livres comme supports de la baraka et comme instruments de perfectionnement spirituel ${ }^{16}$. Nâbulusî, autre akbarien célèbre, défend le même point de vue dans un traité inédit ${ }^{17}$.

9 Mais, dans l'hagiographie, quand on parle d'un saint ummî, c'est toujours d'un saint proprement illettré, ou à tout le moins inculte, qu'il s'agit. Les exemples sont innombrables. Le grand walî berbère Abû Ya'zâ, encore très vénéré aujourd'hui, était de ceux-là. Il n'avait appris du Coran que la Fâtiha et les trois dernières sourates, qui sont parmi les plus courtes. Pour s'entretenir avec ses visiteurs arabophones, il avait besoin d'un interprète. Cela ne l'empêchait pas de déceler miraculeusement les erreurs que pouvait commettre, dans la récitation du Coran, l'imâm qui dirigeait la prière ${ }^{18}$. Abû Ja'far al-Uryabî, le premier maître, très aimé, d'Ibn Arabî, était un paysan andalou qui ne savait 
ni lire ni compter ${ }^{19}$ - et l'on pourrait à ce propos rappeler aussi le cas du shaykh d'un autre saint bien connu, Abû Yazid al-Bistâmî, lequel déclare qu'il dût enseigner à Abû Alî al-Sindî, son initiateur, les règles élémentaires des pratiques rituelles; ou celui, encore, de Abû l-Abbâs al-Qassâb, un des grands maîtres spirituels de la Transoxiane. Dans l'entourage de Muhammad al-Hanafî, prestigieuse figure du soufisme cairote à la fin du XIV siècle, on rencontre aussi un saint ummî, Shams al-dîn Muhammad, surnommé alBâbâ, dont il nous est dit qu'il devint qutb al-zamân, « le Pôle de son époque » quelques instants avant sa mort ${ }^{20}$. Parmi les maîtres de Sha'râni (ob. 1565) - qui est un cas typique d'hagiographe hagiographé - on rencontre aussi deux saints ummî-s, dont il a longuement parlé dans plusieurs de ses oeuvres: Ibrâhim al-Matbûlî et Alî al-Khawwâs, toujours mentionnés avec une affectueuse vénération. Ces personnages - le premier est vendeur de pois chiches, le second marchand d'huile - nous les voyons, dans les pages nombreuses que Sha'ranî leur a consacrées ${ }^{21}$, valider ou invalider des traditions prophétiques d'authenticité disputées, résoudre dans un langage sans apprêt de subtils problèmes doctrinaux, interpréter des versets obscurs qui confondent les exégètes. Ils connaissent les décrets divins, en prédisent les échéances. Auteur savant et fécond, Sha'rânî fait inlassablement appel à l'autorité de ces simples pour trancher les questions qui l'embarrassent.

Un des cas les plus intéressants, parce que les mieux documentés, est celui d'Abd al-Azîz al-Dabbâgh (ob.1129/1717), dont on visite toujours la tombe à Fès, le vendredi, au cimetière de Bâb al-Futûh. Son disciple Ibn al-Mubârak, qui a consigné l'enseignement recueilli de la bouche de son shaykh dans le Kitâb al-Ibrîz ne cache pas son émerveillement devant ce walî qui avait réponse à tout ma'a kawnihi ummiyyan, «bien qu'il fût illettré ». Le récit qu'il rapporte du fath, de l'«illumination » d'Abd al-Azîz al-Dabbâgh ${ }^{22}$ est un document exceptionnel par sa précision phénoménologique et même chronologique (l'événement est daté très exactement du 8 rajab 1125) comme par la simplicité très expressive du langage. Je ne résiste pas au plaisir d'en traduire quelques lignes qui nous font assister à la naissance d'un saint : « Je sortis de chez moi ", raconte l'auteur, « et, par l'intermédiaire d'un des serviteurs charitables, Dieu me gratifia de quatre piécettes de monnaie avec lesquelles j'achetai un poisson que j'apportai chez moi. Ma femme me dit: «Va jusqu'à la mosquée de Sidî Alî b. Hirzîhim et rapporte-moi de l'huile pour faire frire ce poisson ». Je partis donc et voici que, comme j'atteignais Bâb al-Futûh, je fus pris de frissons et de violents tremblements. Puis mon corps (lit. : ma chair) commença à s'agiter abondamment. Je marchais dans cet état qui ne cessait de croître en intensité, jusqu'à la tombe de Sidî Alî b. Hirzîhim. Le hâl (l'état spirituel) devenait de plus en plus fort (...) Je pensai : "Voici la mort, sans aucun doute». Alors sortit de moi comme une vapeur pareille à celle qui monte du couscoussier. J'eus le sentiment que je grandissais au-delà de toute mesure. Les choses de ce monde se dévoilèrent à moi, m'apparaissant comme si elles étaient proches (...) Je vis toutes les mers, et les sept terres, ainsi que les bêtes et autres créatures qu'elles contiennent. Je vis le ciel et il me parût que j'étais au-dessus de lui et que je regardais ce qui s'y trouvait. Et voici que surgit, de toutes les directions à la fois, une immense lumière pareille à l'éclair : elle venait à la fois du haut et du bas, de la droite et de la gauche, devant moi et derrière moi ; et elle produisit en moi un froid glacial qui me fit penser que je mourais. L'idée me vint de me coucher face contre terre afin de ne plus la voir mais, lorsque je m'étendis, mon corps tout entier était devenu oeil : mes yeux voyaient, ma tête voyait et pareillement mon pied et tous mes membres. Mon vêtement lui-même ne pouvait intercepter cette vision de tout mon être. Je compris que, couché ou debout, c'était tout un ». 
11 Cet état (je résume la suite) cesse au bout d'une heure, puis revient, s'en va de nouveau, revient encore, et finit par devenir permanent jusqu'au troisième jour de l'id al-Kabîr de cette même année 1125 où une vision du Prophète stabilise enfin la condition spirituelle d'Abd al-Azîz. Mais, dans le coeur de ce jeune homme ignorant, le fath a fait sourdre toutes les fontaines de la sagesse et il parle désormais de omni re scibili avec l'assurance d'un maître. Ibn al-Mubârak, son élève et futur successeur, est lui, un lettré. Il a même beaucoup lu et ne déteste pas faire étalage de son savoir. C'est en 1125, l'année même du fath que je viens de décrire qu'il rencontre Abd al-Azîz al-Dabbâgh. Pendant quatre ans, jusqu'à la mort du saint, il ne cessera de l'interroger sur d'innombrables sujets: interprétation ésotérique de versets coraniques, authentification de hadith-s suspects, secrets de la science des lettres, description de l'« Assemblée des Saints » (dîwân al-awliyâ) - et bien sûr, règles de vie spirituelle. Fréquentant assidûment l'oeuvre du Shaykh alAkbar, il met parfois son maître à l'épreuve en lui soumettant des passages d'interprétation délicate des écrits d'Ibn Arabî. Dans le style familier qui est le sien, Abd al-Azîz résoud les énigmes et dissipe les contradictions sans recourir à une autre autorité que celle qu'il détient lui-même en vertu de l'infusion divine.

Nous touchons là, avec le cas extrême de ces illettrés dont la science spirituelle n'est pourtant jamais prise en défaut, un point essentiel. On parle souvent du pouvoir des saints. Je voudrais insister sur leur savoir. Ce qui est commun à tous les types d'awliyâ que nous propose l'hagiographie, qu'ils soient humbles ou puissants, fous ou sages, lettrés ou analphabètes, c'est le ilm, la science. (L'emploi de ce mot qui appartient au vocabulaire coranique et correspond à l'un des Noms divins, est, selon Ibn Arabî, préférable à celui de ma'rifa, "connaissance ", que l'on rencontre aussi avec le même sens dans les textes soufis). Leur pouvoir - celui qu'attestent parfois spectaculairement leurs charismes ( karamât) - est celui qui appartient à ceux qui savent. C'est ce qu'exprime un adage très répandu chez les maîtres du soufisme selon lequel «Dieu ne prend jamais comme walî un ignorant ». C'est ce qu'exprime aussi Abd al-Azîz al-Dabbâgh lorsque, regrettant qu'on parle trop des karamât des saints, il dit à son disciple : « Le saint ne se distingue des autres hommes que par une seule chose : à savoir les connaissances et les ouvertures dont Dieu l'a favorisé ». Ibn Arabî va plus loin encore en déclarant: "la science est une des conditions de la sainteté ; la foi (îmân) n'en est pas une condition ${ }^{23}$.

13 Les auteurs qui ont tenté de cerner la notion de sacré en islam se sont généralement intéressés aux emplois de quelques racines verbales: HRM, qui évoque une idée de séparation et d'où procèdent les mots qui, dans le Coran, s'appliquent à l'espace sacré (la Kaaba et la mosquée qui l'entoure) et au temps sacré (quatre mois sur douze) ; QDS (qu'on retrouve dans le nom de Jérusalem: Bayt al-maqdis), qui exprime la transcendance, la pureté et qu'on utilise pour parler de Dieu ou dans les eulogies posthumes de personnages vénérés ${ }^{24} ; B R K$, d'où dérive le terme bien connu de baraka, dont les sens sont divers selon les contextes mais comportent une référence à un influx d'origine surnaturelle qui peut être source de pouvoirs exceptionnels. Il faut, me semble-t-il, ajouter à cette liste la racine GhYB qui dénote l'occulte, le mystère, et constitue une clé indispensable pour comprendre la nature et la fonction de cette catégorie $d$ ' « interprètes du sacré» que représentent les awliyâ.

Ceux qui savent, ceux qui possèdent le ilm, s'appellent en arabe les ulamâ et ce vocable, familier aux non-arabisants eux-mêmes, désigne habituellement les « clercs » - dotés ou non d'une charge officielle - qui, par exemple, s'appliquent à déterminer, par une exégèse laborieuse et controversée du texte révélé, le périmètre exact du "territoire sacré » 
institué par le Coran (tant de kilomètres dans un sens, tant de kilomètres dans l'autre), et les conditions de son accès (les infidèles peuvent-ils y pénétrer? dans l'affirmative, ontils le droit d'y séjourner?). Les awliyâ revendiquent une autre science, immédiate et certaine. Le ghayb, pour eux, n'est pas ghayb: Umar Ibn al-Fârid, marchant sans guide dans le désert pour accomplir son pèlerinage, a la Kaaba sous les yeux tout au long de son voyage. Shâh Walîullâh, pénétrant dans la Mosquée sainte, voit les légions angéliques qui l'entourent. Nombre de saints, arrivant à Médine dans la Rawda (l'espace compris entre la tombe du Prophète et sa chaire et qui, selon une tradition célèbre, est « un des jardins du paradis ») voient le paradis. Là où les ulamâ, au sens usuel de ce mot, s'appuient sur des conjectures, les awliyâ sont forts d'une certitude qui ne contredit pas la légitimité des clercs mais révoque leur monople. Ils peuvent ainsi remplir sans hésiter les blancs de la carte du sacré, convoquer de nouvelles épiphanies.

Cette revendication d'une science qui n'est pas dans les livres, elle se fonde, bien sûr, sur le modèle que propose à sa communauté le "Prophète illettré » (al-nabî al-ummî) qui possède «la science des premiers et des derniers». Elle s'autorise aussi des propos attribués à certains de ces Compagnons, que l'on n'appelait pas encore awliyâ mais qui occupent la place d'honneur dans les grands recueils hagiographiques. C'est Alî b. Abî Tâlib déclarant, en montrant sa poitrine : «Il y a là une science - ah! si seulement je trouvais un homme capable de la porter!». C'est Abû Hurayra, autre Compagnon du Prophète, rapporteur d'innombrables hadîth-s qui confia un jour : «J'ai reçu cinq charges de science. J'ai divulgué deux d'entre elles mais si je divulguais les trois autres, vous me couperiez la gorge !».

Les ulamâ - entendons ici : les clercs, les docteurs - ne s'y sont pas trompés. De toutes les prétentions des awliyâ, la plus insupportable à leurs yeux est précisément de posséder ce ilm bi-lâ wâsita, cette " science sans intermédiaire ». Il n'est que de lire les pages que, dans son Talbîs Iblîs («Les ruses du diable ») ${ }^{25}$, Ibn al-Jawzî (ob. 597/1200) consacre à certaines déclarations de saints illustres, tel Shiblî ou Abû Yazîd al-Bistamî-lequel proclame superbement: "Vous tenez des morts une science morte; je tiens la mienne du Vivant qui ne meurt pas». Un peu plus tard, Ibn Taymiyya (dénonçant ces prétendus saints comme des awliyâa al-shaytân, des "saints de Satan») reprendra en les amplifiant ces critiques auxquelles les wahhâbites, entre autres, font toujours vigoureusement écho aujourd'hui.

Il me paraît y avoir là une bonne raison de ne pas se satisfaire du two-tiered model dont Peter Brown a souligné le simplisme à propos du culte des saints en Occident. Ce schéma réducteur - que les orientalistes n'ont pas inventé : il est déjà présent chez Ibn Taymiyya ou chez Ibn Khaldûn - dans lequel s'affrontent un islam "scripturaire » et un islam "populaire ", me semble n'avoir qu'une valeur heuristique très limitée. Il est beaucoup plus fécond, je crois, d'être attentif, non pas à l'opposition des doctes et des ignorants mais plutôt à la concurrence, à travers l'histoire des sociétés musulmanes, de deux types de science et de deux types de "savants " - les ulamâ et les awliyâ, le "saint illettré " représentant, parmi les seconds, une figure particulièrement exemplaire ${ }^{26}$ en raison de sa proximité du paradigme prophétique, du nabîummî.

Peut-on esquisser un parallèle avec le christianisme latin (où ne manquent pas non plus les saints illettrés : bergères, enfants, rustiques visionnaires...) ? Mon impression, fondée entre autres choses sur l'analyse des procès de canonisation médiévaux que l'on doit à André Vauchez, est que la "science " n'a été prise en considération comme critère de sainteté qu'assez tardivement et qu'on a choisi d'exalter plutôt les vertus et les miracles 
des saints. Peu porté à concéder à des "révélations privées » le droit d'interpréter la parole de Dieu, le magistère ecclésiastique a manifestement une conception fort prudente des dons du Saint-Esprit. La méfiance des censeurs musulmans n'est pas moins vigilante et elle s'inspire de motifs comparables. Il existe toutefois une différence fondamentale : s'il n'y a pas en islam de statut canonique de la sainteté, il n'y a pas non plus de magistère canoniquement irrécusable. Entre ulamâ et awliyâ, la compétition ne peut donc être définitivement close par une procédure disciplinaire ou une mise à l'index. Le âlim, s'il est pourvu d'une fonction officielle, toujours précaire, dispose parfois, pour quelque temps, d'un pouvoir relatif. Il ne détient jamais une autorité absolue. Ce qu'une fatwa a fait, une autre peut le défaire. Entre deux opinions opposées, le croyant est libre de choisir son camp. Et c'est pourquoi, en dépit des protestations des ulamâ - pour qui le discours divin est clos : il ne reste qu'à le gloser - Dieu continue à parler à travers ses awliyâ. Dans un texte de 1963, Ernest Gellner se risquait à dire, à propos des saints de l'islam: «They are on the way out ». Je pense, quant à moi, qu'ils ont un bel avenir devant eux - et que Dieu n'est pas prêt à se taire.

\section{NOTES}

1. Pour les références de ce hadith, voir WENSINCK, Concordance, II, p. 75.

2. Cf. en particulier, R.A. NICHOLSON, Studies in Islamic Mysticism, Cambridge, 1921, ch. II ; A.A. AFIFI, The Mystical Philosophy of Muhyid Din Ibnul Arabî, Cambridge, 1939, ch. II ; L. MASSIGNON, «L'homme parfait en islam et son originalité eschatologique » in Opera Minora, Beyrouth, 1963, I, pp. 107-125 ; H.H. SCHAEDER, Die Islamische Lehre vom Volkommenen Menschen, 2, D.M.G. 79, 1925, pp. 192-268 ; T. IZUTSU, Sufism ant Taoism, Tokyo, 1966, ch. XV-XVII ; R. ARNALDEZ, E12 (s.v. insân kâmil); Masataka TAKESHITA, Ibn Arabî's Theory of the Perfect Man, Tokyo, 1987 (où l'on trouvera une bonne bibliographie) ; M. CHODKIEWICZ, Le Sceau des saints, Paris, 1986, (ch. IV).

3. Futûhât Makkiyya, Le Caire, 1329h, II, p. 391.

4. Fut. II., p. 396

5. Fut. II, p. 446.

6. Selon Ibn Arabî, tous les êtres sont des kalimâtu Llâh (Cf. par ex. Fut., I, p. 336 ; IV, pp. 5, 65, etc.). Sur les références aux principales formulations akbariennes relatives à l'insân kâmil, voir SU'AD HAKÎM, Al-mu'jam al-sûfí, Beyrouth, 1981, pp. 157-168.

7. IBN HANBAL, II, pp. 244, 251, 315 ; BUKHÂRÎ, isti'dhân, 1.

8. Sur le symbolisme du miroir chez Ibn Arabî, cf. Fut. I, p. 112 ; II, pp. 80,116, 131, 134, 290 ; IV, p. 316 ; A.A. AFIFI ed., Fusûs al-hikam, Beyrouth, 1946, I, p. 49.

9. Fut., III, p. 186.

10. Fut. III, p. 188

11. Fut. III, p. 270. J'ai exposé ailleurs (Le Sceau des saints, ch. IV), la signification du thème de l'« héritage" (wirâtha) dans l'hagiologie d'Ibn Arabî, hagiologie qui structure à un degré insoupçonné toutes les théories ultérieures de la sainteté en islam, y compris chez les adversaires d'Ibn Arabî ou chez des auteurs qui ne me semblent pas avoir une connaissance directe de ses oeuvres (voir à ce sujet ma communication The diffusion of Ibn Arabî's doctrine au colloque de Princeton, avril 1989, Transmission of religious Culture in islam.)

12. Julian BALDICK, Mystical Islam, London, 1989, pp. 7-8. 
13. NABHÂNî, Jâmî karamât al-awliyâ, le Caire, 1329/1911.

14. La distribution de ces types dans le temps et l'espace réclamerait une analyse qui malheureusement reste à faire.

15. Sur les sources de cette anecdote voir Fritz MEIER, Die fawâ'ih al-gamâl wa fawâtih al-galâl, Wiesbaden, 1957, pp. 45-46. Selon une autre version, rapportée par Tâsh KABRIZADE, (Miftâh alsa'ada, Hayderabad, 1329, I, pp. 450-451), c'est au contraire au cours de cette retraite ordonnée par Kubrâ que Râzî aurait reçu l'inspiration surnaturelle qui guida ensuite la rédaction de son grand commentaire du Coran.

16. JîLî, Marâtib al-wujûd, Le Caire, s.d., pp. 8-12.

17. NÂBULUSî, Kitâb al-rusukh fi maqâm al-shuyûkh, Ms Berlin We 1631, 189b ss.

18. TÂDILî, Al-tashawwuf ilâ rijâl al-tasawwuf, Rabat, 1984, p. 323.

19. Sur ce personnage, voir Fut., I, pp. 186, 223, 244, 329, 574 ; II, pp. 177, 224, 325, 687 ; II, pp. 208, 532, 539 ; IV, pp. 89, 243, 482, 497, 529 ; et Rûh al-quds, Damas, 1964, notice $\mathrm{n}^{\circ} 1$.

20. Alî AL-BATANÛNÎ, Al-sirr al-safî fî manâqib al-Sultân al-Hanafî, Le Caire, 1306h, II, pp. 74-75.

21. A défaut de pouvoir mentionner tous les passages où apparaissent ces deux personnages, nous renvoyons à SHA'RÂNî, Tabaqât kubrâ, Le Caire, 1954, II, pp. 83-87, 150-169 ; et, du même auteur, à deux ouvrages consacrés à Alî al-khawwâs, le Kitâb durâr al-ghawwâs et le Kitâb al-jawâhir wa l-durar, l'un et l'autre publiés en marge du Kitâb al-Ibrîz d'IBN AL-MUBÂRAK, (voir infra), édition du Caire, s.d., Maktaba Muhammad Alî Subayh.

22. Kitâb al-Ibrîz, Damas, 1984, I, pp. 53-55 (cette édition est préférable à toutes les précédentes et notamment à celle signalée note 21 ).

23. Fut. II, p. 52.

24. Mais c'est de cette racine que les chrétiens de langue arabe ont formé le mot qui désigne les saints.

25. IBN AL-JAWZî. Talbîs Iblîs, ed. Muhammad Munîr, Le Caire, s.d., ch X, pp. 309 sq.

26. Il faut ajouter, pour éviter de substituer un schéma simpliste à un autre, que les saints qui étaient aussi des ulamâ « professionnels » - traditionnalistes, juristes... - sont nombreux tout au long de l'histoire. Citons les noms d'Abd al-Qâdir al-Jîlanî, de Suyûtî, de Shaa'rânî, de Zakariyya al-Ansârî... Constatation qui d'autre part, favorise un peu plus le two-tiered model.

\section{AUTEUR}

\section{MICHEL CHODKIEWICZ}

Michel CHODKIEWICZ est directeur d'études à l'EHESS. 